\title{
Nivolumab in renal cancer
}

\author{
Sabrina Rossetti ${ }^{1}$, Sabrina Chiara Cecere ${ }^{1}$, Carmela Pisano ${ }^{1}$, Gelsomina Iovane ${ }^{1}$, \\ Jole Ventriglia $^{1}$, Imma Paciolla ${ }^{1}$, Marilena Di Napoli ${ }^{1}$
}

\begin{abstract}
Renal cell carcinoma (RCC) is a chemoresistant tumor, characterized by a poor response and lack of survival benefit with conventional cytotoxic agents. Furthermore, the complexity and heterogeneity of RCC limit the biologic targets for novel agents to manage RCC. Expression of the multiple drug resistance (MDR) protein (ATP-binding cassette P-glycoprotein) is increased in RCC, while the Von Hippel Lindau protein is mutated or silenced in approximately half of sporadic RCC, resulting in high levels of hypoxia inhibiting factor (HIF) and the activation of several genes codifying growth factors and growth factors receptors, such as vascular endothelial growth factor (VEGF), platelet-derived growth factor B (PDGF-B), and transforming growth factor alpha (TGF $\alpha$ ). Consequently, immunodrugs targeting pathways related to these proteins have been evaluated for the management of metastatic RCC (mRCC). This review provides an overview of drugs that have been evaluated or are under investigation for the treatment of $\mathrm{mRCC}$, including the multi-tyrosine kinase inhibitors (TKIs) sunitinib, pazopanib, axitinib, the mammalian target of rapamycin (mTOR) inhibitors everolimus and temsirolimus, and nivolumab, which targets the programmed death-1/programmed death-ligand 1 (PD-1/PD-L1) pathway. Promising results have been obtained with nivolumab, alone or in combination with TKIs such as sunitinib and pazopanib, and with ipilimumab, an immune checkpoint inhibitor which acts on the CTLA-4 pathway. Although much progress has been made, research with these and other agents is ongoing, and there is a need to identify makers of response to "tailor" the most suitable therapy, or sequence of therapies, to the individual patient, with the aim of improving outcomes and reducing unneeded toxicity.
\end{abstract}

Key words: renal cell carcinoma, multi-tyrosine kinase inhibitors, mTOR inhibitors, nivolumab, combination therapy

\section{Introduction}

Renal cell carcinoma (RCC) has always been considered a chemoresistant tumor, related to a poor response rate and lack of clear survival benefit from therapy with cytotoxic agents. The biological features underlying this unfavorable clinical outcome involve increased expression of the multiple drug resistance (MDR) protein (ATP-binding cassette P-glycoprotein), which can expel drugs out from tumor cells, reducing their cytotoxic efficacy [1]. Von Hippel Lindau protein is mutated or silenced in about

${ }^{1}$ Division of Medical Oncology, Department of Uro-Gynecological Oncology, Istituto Nazionale Tumori IRCCS

Fondazione G. Pascale, Napoli, Italy.

Correspondence to:

Sabrina Rossetti, MD,

Dipartimento di Uro-Ginecologia Oncologica,

Istituto Nazionale Tumori IRCCS Fondazione G. Pascale,

Via M. Semmola 52, 80131 Napoli, Italy.

Phone: +390815903637 - Fax: +390815903861

E-mail: sabrinarossetti@virgilio.it

CANCER BREAKING NEWS 2017;5(2):12-17

DOI: $10.19156 / \mathrm{cbn} .2017 .0043$ half of sporadic RCC: cancer cells lacking this protein, which usually turns cell replication off in the presence of oxygen, produce high levels of hypoxia inhibiting factor (HIF), then activating the transcription of several genes, almost all codifying growth factors and growth factors receptors, such as vascular endothelial growth factor (VEGF), platelet-derived growth factor B (PDGF$\mathrm{B})$, and transforming growth factor alpha (TGF $\alpha$ ) [2-4]. For these reasons, new agents targeting pathways related to these proteins have been evaluated for the management of metastatic RCC (mRCC). Sunitinib was the first multi-tyrosine kinase inhibitor (TKI) approved in 2006, improving the survival of patients suffering from mRCC compared to interferon alpha (IFN- $\alpha$ ), the standard of care until then [5]. Later, pazopanib and axitinib were approved for first and second line therapy, respectively [6, 7]. These molecules share a similar mechanism of action, which consists of inhibition of several tyrosine kinase receptors (RTKi), mostly VEGFR and PDGFR: these proteins are steadily activated in renal cancer cells, at least as long as new mutations arise in the tumor ge- 
nome, turning off the addiction of cancer cells to these oncogenic pathways, and so developing resistance to these drugs. The phosphatidyl-inositol-3 kinase (PI3K)/ Akt/mammalian target of rapamycin (mTOR) pathway proved to be another molecular target of clinical interest in $\mathrm{mRCC}$. Indeed, mTOR plays a pivotal role in many cellular "devices", as well as regulating the synthesis of HIF and VEGF [8, 9]. mTOR inhibiting agents, such as everolimus and temsirolimus, are currently approved for second line use after TKI therapy failure, and for untreated patients with poor-intermediate prognosis renal tumors, respectively $[10,11]$. Therefore, efforts have been made to "open" new therapeutic scenarios, and the most interesting one represents a kind of "back to the past" journey, to the time when the only therapy tools were the immunostimulating agents, such as interleukin 2 (IL-2) and IFN- $\alpha$, which were, until the TKI era, the standard of care for $\mathrm{mRCC}$ [12].

\section{Return to the past: back to immunotherapy}

Until the middle of the 2000s, few therapeutic options were available in $\mathrm{mRCC}$ management, and these were mostly represented by IL-2 and IFN- $\alpha$. Unfortunately, these agents are burdened by excessive toxicity and limited effectiveness [13, 14]. An interesting attempt was made to "merge" therapy with anti-VEGF agents, of which at that time there was only bevacizumab, a humanized antibody directed against circulating VEGF, and IFN- $\alpha$, but even this combination therapy did not significantly improve survival for patients with mRCC [15]. Nevertheless, a renewed interest in this pharmacological approach arose with the advent of a new class of immunotherapy agents, targeting programmed death-1/ programmed death-ligand 1 pathway (PD-1/PD-L1). In fact, as well as melanoma, renal cancer shows a massive infiltration of immune cells, consisting mostly of $\mathrm{T}$ cells, natural killer (NK) and dendritic cells: these cells cannot exert their functions because of being induced to a state of anergy [16], and PD-1/PD-L1 was shown to have a preeminent role in this. As stated, melanoma and RCC share similar features, such as great infiltration of immune cells and extensive chemoresistance, and these reasons underlie the clinical interest in drugs targeting immune checkpoints. CD4+/CD8+ T cells, NK cells, B cells and monocytes express PD-1 on induction of several cytokines, such as IL-2, IL-7, IL-15 and IL-21 [17, 18], and patients whose tumors contain PD-1 positive tumorinfiltrating lymphocytes (TILs) having a poorer outcome than patients with PD-1 negative lymphocytes [19]. On the other hand, PD-1 ligands, namely PD-L1 (also called B7-H1), and PD-L2 (also known as B7-DC), are expressed not only by several immune-regulating cells, but also by cancer cells: PD-L1 expression has been detected in up to two-thirds of clear cell renal carcinomas, with significantly worse cancer-specific survival of patients suffering from PD-L1 overexpressing disease. Furthermore, PD-L1-enriched TILs infiltrating primary RCC correlate with advanced stage and shorter progression-free survival (PFS) [20, 21], thus providing the biological foundations for PD-1/PD-L1 blockade in order to increase the reactivity of the host immune system against the tumor.

\section{Targeting the PD-1/PD-L1 pathway}

Nivolumab is a fully humanized antibody directed against PD-1 which acts by breaking the PD-1/PD-L1 interaction, so preventing the latter from "turning off" TILs and other immune cells, which otherwise would be induced into a state of anergy [22, 23]. Because of its high affinity to the target, nivolumab rapidly disappears from the bloodstream, nevertheless persisting in occupying PD-1 receptors for up to 3 months after infusion [24]. Since the catabolic pathway of nivolumab is the same as that of regular immunoglobulin $\mathrm{G}$ ( $\mathrm{IgG}$ ), no dose adjustment is needed in patients with mild or moderate renal impairment or mild hepatic impairment; limited data are available from patients with severe renal or moderateto-severe liver impairment [25]. After the encouraging results of phase I trials recruiting patients with metastatic melanoma, non-small cell lung carcinoma and RCC $[26,27]$, a phase II trial showed median PFS and overall survival (OS) of 4.2 and 24.7 months, respectively, for patients with mRCC treated with nivolumab at a dose of $10 \mathrm{mg} / \mathrm{kg}$, after having received no more than three previous therapy lines with at least one VEGFR TKI [28]. More recently, the CheckMate 025 trial, an open-label, phase III study, compared nivolumab $3 \mathrm{mg} / \mathrm{kg}$ every two weeks with everolimus $10 \mathrm{mg} /$ day in patients with mRCC who progressed on at least one TKI therapy line (no more than three previous treatments). Nivolumabtreated patients had a longer OS compared with everolimus (median OS 25 vs 19.6 months; hazard ratio [HR] for risk of death 0.73 ), with no difference in terms of PFS (4.6 vs 4.4 months). Of note, a subgroup analysis of patients with OS and PFS longer than 6 months revealed a statistically significant difference in PFS between patients who received nivolumab and those who took oral everolimus: nivolumab median PFS 15.6 months vs 11.7 months for the everolimus group (HR for progression risk 0.64), irrespective of PD-L1 expression [29]. At the 2016 Genitourinary Cancers Symposium, authors presented a subgroup analysis further supporting the use of nivolumab as a new standard of care in patients with 
previously treated $\mathrm{mRCC}$, regardless of Memorial Sloan Kettering Cancer Center (MSKCC) and International Metastatic Renal Cell Carcinoma Database Consortium (IMRCC) prognostic score, number or duration of prior therapies (sunitinib, pazopanib, or IL-2 therapy), age and sex, with a lower incidence of grade 3 or 4 treatmentrelated adverse events (AEs). Of interest, patients with poor MSKCC risk seem to benefit more from nivolumab than everolimus (HR $0.48,95 \%$ confidence interval 0.32-0.70) [30].

In a large expanded access program (EAP) that enrolled 389 patients across 95 Italian sites, clear-cell histology was the most common ( $92 \%$ of cases), and half of the patients had bone metastases. Only 18 patients $(5 \%)$ discontinued treatment because of an AE. The disease control rate (DCR) was $48 \%$ (17\% objective response; one complete and 66 partial responses), while $121 \mathrm{pa}-$ tients $(31 \%)$ had stable disease [31]. Brain metastases occur quite rarely in renal cancer (approximately 10\% of mRCC) and the ability of monoclonal antibodies to cross the blood-brain barrier (BBB) is a hot topic. Furthermore, the administration of steroids often becomes necessary due to neurologic symptoms triggered by metastatic lesions and peripheral edema, thereby decreasing the efficacy of immunodrugs. Fifty-three patients with $\mathrm{mRCC}$ and brain metastases from GETUG-AFU 26 (Nivoren) Study were treated with nivolumab after having received previous mTOR inhibitor as systemic therapy. Five had had previous brain surgery and 17 brain radiation. Forty-four patients were evaluated for response on brain metastases, with 10 achieving an objective response (response rate $23 \%$ ), while 21 had locally progressive disease. Nevertheless, neurologic symptoms required steroids administration in 15 patients [32].

\section{Combination therapy: can efficacy be improved?}

As has been shown in other malignancies, therapies combining multiple drugs have been shown to be more effective, resulting in a synergistic, rather than additive, effect compared with monotherapy alone, although with an associated increase in the incidence of AEs. In this regard, nivolumab has been administered both in combination with TKIs, such sunitinib, and pazopanib and with ipilimumab, another immunotherapy agent acting on the CTLA-4 pathway. The biological rationale underlying the association of immune checkpoint inhibitors with TKIs rely on the ability of the latter to modulate the immune microenvironment, with a decrement of circulating regulatory $\mathrm{T}$ cells (T-regs) and myeloid-derived suppressor cells $[33,34]$. In a first-line treatment-naïve setting, the nivolumab plus pazopanib combination was associated with dose-limiting liver toxicity, while the nivolumab plus sunitinib combination was well tolerated to a higher nivolumab dose. Although both drugs proved to be effective when combined with nivolumab in terms of encouraging antitumor activity, data from randomized trials are needed to evaluate the efficacy of combination versus monotherapy [35].

As expected, further studies were designed to assess combinations of TKIs and other anti-PD-1 agents, such as axitinib plus pembrolizumab [36] or axitinib plus anti PD-L1 agents, such as avelumab [37]. These latter agents, when administered together, did not show an increased incidence of AEs compared with single agent therapy, with two deaths during the study period (1 disease-related and 1 treatment-related). AEs led to discontinuation of avelumab in 5 patients $(9.1 \%)$ and axitinib in 4 patients (7.3\%). The overall response rate was $54.5 \%$, consisting of two complete responses and 28 partial responses [37].

As already noted, multiple immune checkpoint inhibition is also an attractive option. The CheckMate 016 phase I trial combined nivolumab with ipilimumab in patients with mRCC with encouraging results [38]. Therefore, this combination is under evaluation compared with sunitinib monotherapy in the first-line setting in a phase III trial (CheckMate 214; ClinicalTrials.gov identifier: NCT02231749). Combination of antibodies targeting PD-L1 and circulating VEGF have also been investigated. Atezolizumab/bevacizumab showed a decreased risk of disease progression in previously untreated patients with $\mathrm{mRCC}$, although not statistically significant, compared to sunitinib. Of note, the HR for PFS decreased concomitantly with the percentage of PD-L1-expressing cells (HR 0.87 for tumors with $\geq 1 \%$ and $<5 \%$ PD-L1 expressing cells, HR 0.5 for $\geq 5 \%$ and $<10 \%$ and HR 0.23 for tumors expressing PD-L1 in $\geq 10 \%$ ). Finally, there was a statistically significant risk reduction for disease progression in patients with high T-effector count [39].

\section{How can the best drug \\ for the best patient be chosen?}

Unlike some malignancies, for which we have the right drug for the right neoplastic aberration, RCC lacks a singular oncogene addiction that can form the target for immunotherapy agents. Nevertheless, the choice of patients who could take the most advantage of immunodrugs is definitely an unmet need. Already known from the use of IFN- $\alpha$ and IL-2 is that patients treated with these immunostimulating agents benefit from a longer OS after 
undergoing nephrectomy [40], although some evidence suggests that surgical removal of the primary tumor may result in a decrease of PD-1 and PDL-1 expression, thus decreasing the targets for immunodrugs [41, 42]. Therefore, clinicians are looking for possible predictive markers of response, in order to "tailor" the most suitable therapy for each patient, preventing unneeded toxicity and reducing costs. PD-L1 expression on cancer cells seems to affect outcome and response to PD-1 pathway inhibitors [43, 44], but, importantly, the activity of TKIs (either pazopanib or sunitinib) was reduced in patients whose tumors overexpressed PD-L1, resulting in significantly shorter OS [45]. In addition to PD-L1, the presence of $>300$ intratumoral CD8 $+\mathrm{T}$ cells also proved to be a marker of poor prognosis [45]. This has been confirmed by other authors. The detection of high counts of $\mathrm{CD} 8+\mathrm{T}$ cells close to the neoplastic invasion margin, along with the presence of immune cells expressing PD-1 and cancer cells expressing PD-L1, is evidence of immune-editing that the cancer exerts on the microenvironment, "turning off" $\mathrm{T}$ cells by inducing them into a state of anergy [46], and thus preventing them from properly triggering an immune response. In addition to PD-1/ PD-L1 expression and infiltration of tumor by T cells, levels of genomic aberrations correlate with response to immunodrugs. Indeed, the more mutations cancer cells accumulate in their genomes, the more they are likely to express aberrant, and therefore immunogenic, proteins. Loss of DNA mismatch repair genes, such as MLH1 and MSH2, makes RCC a genetically unstable malignancy, leading to an increased PD-L1 expression on RCC cells and concomitant PD-1 expression by TILs [47], but further clinical evidence is needed to support a strong correlation between these features and effectiveness of PD-1/ PD-L1 antibodies. Histology does not seem to help us in recognizing patients more likely to benefit from immunodrugs; indeed, nivolumab has also proved to be effective in non-clear cell renal cancers, such as papillary histotype, with a $29 \%$ partial response rate and $19 \%$ of stable disease [48].

\section{Is immunotherapy more toxic than TKIs?}

The excellent safety profile of nivolumab in patients with advanced RCC has been highlighted in many studies [24, $27,28]$, with a lower incidence of treatment-related side effects than everolimus [29]. In the CheckMate 025 trial, almost $80 \%$ of patients treated with nivolumab and $88 \%$ of everolimus patients experienced AEs of any grade, although grade 3 or 4 AEs were reported in only in 19\% and $37 \%$ of patients, respectively, with the most common AEs consisting of fatigue (33\%), nausea (14\%), and pruritus (14\%) among patients undergoing nivolumab, and fatigue (34\%), stomatitis (29\%), and anemia (24\%) among patients who received everolimus. This led to a slightly greater percentage of drug discontinuation with everolimus compared to nivolumab (13\% vs $8 \%)$. Given its distinguishing mechanism of action, nivolumab is associated with immune-mediated adverse reactions which, although rare, include immune-mediated pneumonitis, colitis, hepatitis, nephritis, and thyroiditis $[25,28]$. An intriguing issue is obviously the change of therapeutic index in combination strategies. Adding a VEGF-TKI to nivolumab resulted in an increased incidence of grade 3-4 AEs $(82 \%$ of nivolumab plus sunitinib treated patients) with alanine aminotransferase (ALT) elevation and hypertension in $18 \%$. Toxicity-related therapy discontinuation was necessary in one third of patients. The combination of nivolumab and pazopanib led to grade 3-4 liver toxicity in $20 \%$ of subjects, with ALT and aspartate aminotransferase (AST) elevation which, in addition to grade 3-4 fatigue in $15 \%$, was the reason for stopping recruitment into this arm [35]. Similar results were found with the pazopanib/pembrolizumab combination, with concomitant therapy burdened by significant hepatotoxicity. Conversely, the sequential schedule of pazopanib for nine weeks, followed by pembrolizumab plus pazopanib, showed reduced hepatotoxicity, with preliminary signs of efficacy but overall limited tolerability [49].

For patients undergoing nivolumab/ipilimumab in combination, grade 3-4 AEs were reported in $43 \%$ of patients, mostly elevated lipase and ALT (16\% and 11\% respectively), diarrhea (9\%), and colitis (5\%). Treatment discontinuation was necessary for $16 \%$ of patients [38].

\section{Conclusions}

Since the early 1990s much progress has been made in the understanding of the biology of RCC, coupled with outstanding results in terms of survival and quality of life. Combination therapy in this malignancy is a hot topic. Acting on multiple targets, both immune checkpoints and VEGF/mTOR pathways could be an effective strategy in order to better (and for longer) control disease progression, although the cost to pay for these improvements is an increased incidence of AEs. Furthermore, the importance of the correct sequencing cannot be disregarded, which will need to be defined by future clinical trials. The complexity and heterogeneity of the cancer slow down our progress, but also provide new tools and therapy options. Therapy "tailored" to fit individual characteristics, both of the host and the cancer, represents a real challenge, and future perspectives will see 
proper use of these opportunities, by identifying patients most likely to benefit from each specific drug.

\section{Acknowledgments}

The Authors thank Ray Hill, an independent medical writer, who provided native English editing and journal

\section{References}

1. Hartmann JT, Bokemeyer C. Chemotherapy for renal cell carcinoma. Anticancer Res 1999;19(2C):1541-3.

2. Kaelin WG, Jr. The von Hippel-Lindau tumor suppressor gene and kidney cancer. Clin Cancer Res 2004;10(18 Pt 2): 6290S-5S.

3. Arjumand W, Sultana S. Role of VHL gene mutation in human renal cell carcinoma. Tumour Biol 2012;33(1):9-16.

4. Cowey CL Rathmell WK. VHL gene mutations in renal cell carcinoma: role as a biomarker of disease outcome and drug efficacy. Curr Oncol Rep 2009;11(2):94-101.

5. Motzer RJ, Hutson TE, Tomczak P et al. Sunitinib versus interferon alfa in metastatic renal-cell carcinoma. N Engl J Med 2007;356(2):115-24.

6. Escudier B, Gore M. Axitinib for the management of metastatic renal cell carcinoma. Drugs R D 2011;11(2):113-26.

7. Motzer RJ, Hutson TE, Cella D et al. Pazopanib versus sunitinib in metastatic renal-cell carcinoma. N Engl J Med 2013;369(8):722-31.

8. Battelli C, Cho DC. mTOR inhibitors in renal cell carcinoma. Therapy 2011;8(4):359-67.

9. Voss MH, Molina AM, Motzer RJ. mTOR Inhibitors in advanced renal cell carcinoma. Hematol Oncol Clin North Am 2011;25(4):835-52.

10. Motzer RJ, Escudier B, Oudard S. Phase 3 trial of everolimus for metastatic renal cell carcinoma: final results and analysis of prognostic factors. Cancer 2010;116(18):4256-65.

11. Afshar M, Pascoe J, Whitmarsh S. Temsirolimus for patients with metastatic renal cell carcinoma: outcomes in patients receiving temsirolimus within a compassionate use program in a tertiary referral center. Drug Des Devel Ther 2015;9:13-9.

12. Itsumi M, Tatsugami K. Immunotherapy for renal cell carcinoma. Clin Dev Immunol 2010;2010:284581.

13. Minasian LM, Motzer RJ, Gluck L et al. Interferon alfa-2a in advanced renal cell carcinoma: treatment results and survival in 159 patients with long-term follow-up. J Clin Oncol 1993;11(7):1368-75.

14. Margolin KA. Interleukin-2 in the treatment of renal cancer. Semin Oncol 2000;27(2):194-203.

15. Escudier B, Bellmunt J, Négrier S. Phase III trial of bevacizumab plus interferon alfa-2a in patients with metastatic renal cell carcinoma (AVOREN): final analysis of overall survival. J Clin Oncol 2010;28(13):2144-50.

16. Noessner E, Brech D, Mendler AN et al. Intratumoral alterations of dendritic-cell differentiation and CD8(+) T-cell anergy are immune escape mechanisms of clear cell renal cell carcinoma. Oncoimmunology 2012;1(8):1451-3.

17. Keir M, Butte M, Freeman G et al. PD-1 and its ligands in tolerance and immunity. Annu Rev Immunol 2008 26:677-704. styling on behalf of HPS. This editorial assistance was funded by PharmaMar, Spain.

\section{Conflicts of Interest}

The Authors declare there are no conflicts of interest in relation to this article.

18. Keir ME, Francisco LM, Sharpe AH. PD-1 and its ligands in T-cell immunity. Curr Opin Immunol 2007;19(3):309-14.

19. Thompson RH, Dong H, Lohse CM et al. PD-1 is expressed by tumor-infiltrating immune cells and is associated with poor outcome for patients with renal cell carcinoma. Clin Cancer Res 2007;13(6):1757-61.

20. Thompson R, Gillett M, Cheville J et al. Costimulatory B7-H1 in renal cell carcinoma patients: Indicator of tumor aggressiveness and potential therapeutic target. Proc Natl Acad Sci USA 2004;101(49):17174-9.

21. Thompson RH, Gillett MD, Cheville JC et al. Costimulatory molecule B7-H1 in primary and metastatic clear cell renal cell carcinoma. Cancer 2005;104(10):2084-91.

22. Berman D, Korman A, Peck R et al. The development of immunomodulatory monoclonal antibodies as a new therapeutic modality for cancer: The Bristol-Myers Squibb experience. Pharmacol Ther 2015;148:132-53.

23. Wang $C$, Thudium $K$, Han et al. In vitro characterization of the anti-PD-1 antibody nivolumab, BMS-936558, and in vivo toxicology in non-human primates. Cancer Immunol Res 2014;2(9):846-56.

24. Brahmer JR, Drake CG, Wollner I et al. Phase I study of single-agent anti-programmed death-1 (MDX-1106) in refractory solid tumors: Safety, clinical activity, pharmacodynamics, and immunologic correlates. J Clin Oncol 2010;28(19):3167-75.

25. European Medicines Agency (EMA) 2017. OPDIVO (nivolumab $10 \mathrm{mg} / \mathrm{mL}$ ): Summary of product characteristics. Available from: http://www.ema.europa.eu/

26. Lipson EJ, Sharfman WH, Drake CG et al. Durable cancer regression off-treatment and effective reinduction therapy with an anti-PD-1 antibody. Clin Cancer Res 2013;19(2):462-8.

27. Topalian SL, Hodi FS, Brahmer JR et al. Safety, activity, and immune correlates of anti-PD-1 antibody in cancer. N Engl J Med 2012;366(26):2443-54.

28. Motzer RJ, Rini BI, McDermott DF et al. Nivolumab for metastatic renal cell carcinoma: results of a randomized phase II trial. J Clin Oncol 2015;33(13):1430-7.

29. Motzer RJ, Escudier B, McDermott DF et al. Nivolum$\mathrm{ab}$ versus everolimus in advanced renal-cell carcinoma. N Engl J Med 2015;373(19):1803-13.

30. Escudier B, Sharma P, McDermott DF et al. CheckMate 025 randomized phase 3 study: Outcomes by key baseline factors and prior therapy for nivolumab versus everolimus in advanced renal cell carcinoma. Eur Urol 2017. pii:S0302-2838(17)30099-4.

31. De Giorgi U, Scagliarini S, Basso U. Safety and efficacy of nivolumab for metastatic renal cell carcinoma (mRCC): Real world data from an Italian expanded access program 
(EAP) [Abstract]. J Clin Oncol 2017;35(15 suppl):4577.

32. Escudier BJ, Chabaud S, Borchiellini D et al. Efficacy and safety of nivolumab in patients with metastatic renal cell carcinoma (mRCC) and brain metastases: Preliminary results from the GETUG-AFU 26 (Nivoren) study [Abstract]. J Clin Oncol 2017;35(15_suppl):4563.

33. Ko JS, Rayman P, Ireland J et al. Direct and differential suppression of myeloid-derived suppressor cell subsets by sunitinib is compartmentally constrained. Cancer Res 2010;70(9):3526-36.

34. Ko JS, Zea AH, Rini BI et al. Sunitinib mediates reversal of myeloid-derived suppressor cell accumulation in renal cell carcinoma patients. Clin Cancer Res 2009;15(6):2148-57.

35. Amin A, Plimack E, Infante J et al. Nivolumab (anti-PD1 ;BMS-936558, ONO-4538) in combination with sunitinib or pazopanib in patients with metastatic renal cell carcinoma (mRCC) [Abstract]. J Clin Oncol 2014;32(15_Suppl):5010.

36. Atkins M, Gupta S, Choueiri T et al. Phase Ib dose-finding study of axitinib plus pembrolizumab in treatment-naïve patients with advanced renal cell carcinoma. J Immunother Cancer 2015;3(Suppl. 2):P353.

37. Choueiri TK, Larkin JMG, Oya M et al. First-line avelumab + axitinib therapy in patients (pts) with advanced renal cell carcinoma (aRCC): Results from a phase Ib trial [Abstract]. J Clin Oncol 2017;35(15_suppl):4504.

38. Hammers HJ, Plimack ER, Infante JR et al. Safety and efficacy of nivolumab in combination with ipilimumab in metastatic renal cell carcinoma: The CheckMate 016 study. J Clin Oncol 2017:JCO2016721985. [Epub ahead of print]

39. McDermott DF, Atkins MB, Motzer RJ et al. A phase II study of atezolizumab (atezo) with or without bevacizumab (bev) versus sunitinib (sun) in untreated metastatic renal cell carcinoma (mRCC) patients (pts) [Abstract]. J Clin Oncol 2017;35(6_suppl):431.

40. Flanigan RC, Salmon SE, Blumenstein BA et al. Nephrectomy followed by interferon alfa- $2 \mathrm{~b}$ compared with inter- feron alfa- $2 \mathrm{~b}$ alone for metastatic renal-cell cancer. N Engl J Med 2001;345(23):1655-9.

41. Jilaveanu LB, Shuch B, Zito CR et al. PD-L1 expression in clear cell renal cell carcinoma: An analysis of nephrectomy and sites of metastases. J Cancer 2014;5(3):166-72.

42. MacFarlane AWt, Jillab M, Plimack ER et al. PD-1 expression on peripheral blood cells increases with stage in renal cell carcinoma patients and is rapidly reduced after surgical tumor resection. Cancer Immunol Res 2014;2(4):320-31.

43. Taube JM, Klein A, Brahmer JR et al. Association of PD-1, PD-1 ligands, and other features of the tumor immune microenvironment with response to anti-PD-1 therapy. Clin Cancer Res 2014;20(19):5064-74.

44. Choueiri T, Fishman M, Escudier B et al, Immunomodulatory activity of nivolumab in metastatic renal cell carcinoma. Clin Cancer Res 2016;22(22):5461-71.

45. Choueiri TK, Figueroa DJ, Fay AP et al. Correlation of PDL1 tumor expression and treatment outcomes in patients with renal cell carcinoma receiving sunitinib or pazopanib: results from COMPARZ, a randomized controlled trial. Clin Cancer Res 2015;21(5):1071-7.

46. Tumeh PC, Harview CL, Yearley JH et al. PD-1 blockade induces responses by inhibiting adaptive immune resistance. Nature 2014;515(7528):568-71.

47. Joseph RW, Kapur P, Serie D et al. Association of loss of BAP1 expression in cell renal cell carcinomas with PDL1 expression [Abstract]. J Clin Oncol 2014;32(15_sup$\mathrm{pl}): 4530$.

48. Koshkin VS, Barata PC, Vogelzang NJ et al. Nivolumab treatment for patients with non-clear cell renal cell carcinoma: A multicenter retrospective analysis [Abstract]. J Clin Oncol 2017;35(15_suppl):4586.

49. Chowdhury S, McDermott DF, Voss MH et al. A phase I/II study to assess the safety and efficacy of pazopanib (PAZ) and pembrolizumab (PEM) in patients (pts) with advanced renal cell carcinoma (aRCC) [Abstract]. J Clin Oncol 2017;35(15_suppl):4506. 